Interviews

\title{
An admissions tutor's perspective on the multiple mini interview
}

What this type of interview reveals and how they are assessed

- By: Philip Adds

In the past five years, the number of medical schools in the United Kingdom moving from panel interviews to multiple mini interviews (MMls) to assess applicants has increased. At present, roughly half of UK medical schools use MMI style interviews. One of the reasons for this is that MMls are reported to be a better predictor of future academic success and professional behaviour than panel interviews. [1] [2] [3] [4] [5]

The format of a MMI differs from a panel interview in that instead of being asked questions from one panel of interviewers, applicants rotate around a series of stations that cover different questions posed by a variety of interviewers or assessors, including admissions staff, practising doctors, medical students, and expert patients. The interviewer at each station does not know anything about the applicant apart from his or her name, and does not hear their response to any of the other questions. One criticism of panel interviews has been that the presence of a senior member of staff can lead to bias, with panel members likely to go along with the chair's view of an applicant. I was a junior member on such a panel, and remember waiting for other panellists to give their scores for candidates I was unsure about before making my own views known. MMls are also often seen as a fairer way of assessing candidates, with each applicant given the same question at each station, and the same allocation of time to answer each question.

$\underline{\text { Top }}$

\section{What are MMls designed to test?}

Each MMI station assesses at least one of the key competencies described by Patterson and colleagues: academic ability, empathy, insight and integrity, teamwork, communication skills, effective learning style, initiative and resilience, organisation, and problem solving.[6] At some stations you will be assessed on more than one competency-for example, communications skills are assessed at every station. Although each medical school will be different, stations are likely to include:

- Ethical scenario

- Interaction with an actor or patient - for example, delivering bad news

- Discussion of recent medical research 
- Problem solving

- Discussion of work experience

- Equality and diversity issues — such as your experiences of working with people from different cultures

- Examples of teamwork and/or leadership Answering MMI questions requires a different approach from answering questions at a panel interview. At some stations, you might be asked questions about what type of work experience you have undertaken, or asked to give examples of leadership experience. At other stations, you might be required to actively demonstrate your problem solving abilities, or that you are a logical thinker, or empathetic. At these types of stations, the underlying principle is to show, rather than tell, the interviewer that you are competent in these areas.

$\underline{\text { Top }}$

\section{How are MMls assessed?}

The type of stations, the amount of time you have to prepare and answer, and the marking scheme will differ between medical schools.

At St George's, University of London, if you achieve the minimum academic grades for entry (including the required target in the UK Clinical Aptitude Test (UKCAT) or the Graduate Australian Medical School Admissions Test (GAMSAT) entrance exams), you will be invited for an interview. We then operate a level playing field approach, so that the decision on whether to offer a place is based solely on your performance at the MMI. Your personal statement does not directly influence this decision, but will be checked before an offer is made. Some medical schools use a weighting system based on your personal statement and/or work experience record, so it is worth double checking their policies on the Student BMJ's medical school profile pages (http://medschoolselector.student.bmj.com/ ).

The marking scheme at St George's is out of five marks on each station (with zero the lowest and five the highest mark). At the end of the interview, all of your scores are added together and if you meet the cut-off score you will be offered a place. This cut-off score changes from year to year.

In addition to this numerical score, applicants are given a global rating of "excellent," "acceptable," or "unacceptable," depending on their overall performance. A "red flag" can be given by interviewers if an applicant says something outrageous or inappropriate during their answer. Any applicant who scores two or below, or an "unacceptable," or has a "red flag," will be automatically referred to the admissions tutor before an offer is made. 


\section{What are admissions tutors looking for in an applicant at a MMI?}

By the interview stage, we already know that you have the academic potential to succeed in medicine. At this point, we are looking for people who have the potential to succeed at medical school-more specifically, those who will succeed at our medical school-by showing that they have the qualities needed to become a good doctor, as outlined in the General Medical Council's Good Medical Practice.[7]

An assessor will be looking at several factors when grading an applicant at his or her station. Firstly, has the applicant answered the question? This may sound obvious, but in many cases they haven't. Secondly, is their answer rehearsed? Trotting out a rehearsed answer can sound false, and you may end up answering a different question from the one asked. For example, every applicant is likely to be asked a question along the lines of, "Why do you want to be a doctor?" Many applicants perform poorly at this question, partly because it is so predictable. If you have a prepared answer at the ready, but it is really for a slightly different question, you will be marked down. Always be yourself-if you are reciting a prepared answer, the interviewer is not seeing the real you. Show your interviewer who you are, what you can do, and why you, more than all the other candidates, are the one that should be picked.

Ultimately, assessors want to see a candidate who is passionate about their chosen career, who has the ability to keep a clear head, to think rationally under pressure, to be aware of developments and controversies in medicine, who has insights into their strengths and shortcomings, and, moreover, who can communicate all this in a clear and articulate manner.

\section{$\underline{\text { Top }}$}

Box 1: Do's and don'ts for MMIs

Top

Do

- Practise speaking for five minutes on a particular topic-it's longer than you think

- Arrive on time and dress smartly - it shows that you are taking the interview seriously

- Check if you are expected to introduce yourself at each station. This will vary between medical schools.

- Answer the question - obvious tip, but often forgotten

- Ask the assessor to repeat the question if you did not hear or understand it Top 
- Rehearse your answers too much. Be prepared, but don't memorise answers word for word from interview preparation resources

- Expect the assessor to explain the question or prompt you. Every candidate gets asked the question in the same way

- Rush your answers. Take a few seconds before replying to settle your nerves and organise your thoughts so that you can answer in a more confident and calm manner

- Speak so loudly that all the other applicants can hear your answer. You don't want them to pinch your ideas

- Panic if you think you have done poorly at one station. Your final mark will be an aggregate, so one station where you don't do so well doesn't matter. Clear your mind and get on with the next one

Philip Adds, admissions tutor

St George's, University of London, UK

Competing interests: None declared.

Provenance and peer review: Commissioned; not externally peer reviewed.

\section{References}

1. Eva KW, Reiter HI, Rosenfeld J, et al. The ability of the multiple miniinterview to predict pre-clerkship performance in medical school. Acad Med 2004;79:40-2 doi:10.1097/00001888-200410001-00012.

2. Eva KW, Reiter HI, Trinh K, Wasi P, Rosenfeld J, Norman GR. Predictive validity of the multiple mini-interview for selecting medical trainees. Med Educ 2009;43:767-75. doi:10.1111/j.13652923.2009.03407.x pmid:19659490.

3. O'Brien A, Harvey J, Shannon M, Lewis K, Valencia O. A comparison of multiple mini-interviews and structured interviews in a UK setting. Med Teach 2011;33:397402. doi:10.3109/0142159X.2010.541532 pmid:21355692.

4. Husbands A, Dowell J. Predictive validity of the Dundee multiple miniinterview. Med Educ 2013;47:71725. doi:10.1111/medu. 12193 pmid:23746161.

5. Knorr M, Hissbach J. Multiple mini-interviews: same concept, different approaches. Med Educ2014;48:115775. doi:10.1111/medu.12535 pmid:25413910.

6. Patterson F, Tavabie A, Denney M, et al. A new competency model for general practice: implications for selection, training, and careers. $\mathrm{Br} \mathrm{J} \mathrm{Gen}$ Pract 2013;63:331-8. doi:10.3399/bjgp13X667196 pmid:23643231.

7. General Medical Council. Good medical practice. 2013. www.gmcuk.org/guidance/good_medical_practice.asp. 